\section{An unusual abdominal pain in the emergency department: a delayed presentation of diaphragmatic injury}

\author{
Ciro Paolillo, Valentina Siragusa \\ Emergency Department, Udine University \\ Hospital, Udine, Italy
}

\begin{abstract}
We present a case of delayed traumatic diaphragma rupture. The patient complained of a vague abdominal pain and left shoulder pain. Only the relevance of a remote trauma aroused the suspicion.
\end{abstract}

\section{Introduction}

The most common cause of diaphragmatic injury is a direct blow to the upper abdomen or lower chest. It is unusual after penetrating injuries such as a knife or bullet wound. The forces transmitted to the abdomen by blunt trauma can create a pressure gradient across the diaphragm of up to $1000 \mathrm{~cm} \mathrm{H}_{2} \mathrm{O}$, which may be sufficient to cause diaphragmatic injury and hence herniation of abdominal contents.

The left side of the diaphragm is more prone to injury because it is not protected by the liver. Kelly ${ }^{1}$ reported that right sided ruptures are less frequent because they are associated with higher prehospital mortality; this likelihood decreases the number of surviving trauma victims with right sided diaphragmatic injuries. Herniated organs generally include stomach, small bowel, colon, spleen and liver.

In 1974, Grimes ${ }^{2}$ described the three phases of the rupture of the diaphragm based on time, after forty years this is still the most useful classification. The acute phase starts at the time of injury. Blunt, high velocity trauma increases the gradient between the abdominal compartment and the thorax, causing avulsion of the diaphragm from its point of attachment. If the diaphragmatic injury is not recognised during the immediate post traumatic period, then the patient progresses into the latent phase, characterized by intermittent visceral herniation of any abdominal structure due to the defect. Sometimes, the patient remains asymptomatic, and, sometimes patients complain of intermittent or nonspecific symptoms, nausea, vomiting, belching and vague postprandial abdominal pain that is better with sitting or standing. The obstructive phase begins when the herniated abdominal organs becomes incarcerated. The patient complains of dyspnea, acute abdominal pain and vomiting. If not immediately treated the consequences can be catastrophic.

Despite technological advances, a delay in diagnosis is common. Wallack in $2010^{3}$ reported that $30-50 \%$ of traumatic diaphragmatic ruptures are missed on initial presentation.

\section{Case Report}

A 62-year-old man, presented to the ED complaining of abdominal pain. The patient was afebrile, his blood pressure was 120/80, respiratory rate was 18 , heart rate was 90 and the oxygen saturation at room's air was $95 \%$. He reported a moderate pain in his left abdomen. The abdomen was soft, not were tender nor distended. Physical examination and ECG were normal. The biochemistry revealed a mild increase of white blood cells $(11,000$ cells/mmc) and C-Reactive Protein $7 \mathrm{mg} / \mathrm{dL}$. Hepatic, pancreatic and renal parameters where within the normal limits. Clinical ultrasound evidenced a normal abdominal aorta and a left renal image, no free fluid was present. It looked like a case of aspecific abdominal pain. After $3 \mathrm{mg}$ of morphine the patient was brought in Radiology. Antero-posterior (AP) and lateral chest X-ray showed an air fluid level that obscured the left diaphragm (Figures 1 and 2). There was not mediastinal shift. The abdominal X-ray was normal. After a couple of hours the patient felt better and his pain has lowered. The ongoing physician reviewed the past clinical history. The patient reported a motor vehicle accident dating four months. He had thoracoabdominal trauma with a small pneumothorax (PNX) and several broken ribs on the left side. At the time thoracoabdominal computerised tomography (CT) denied a diaphragmatic rupture. He then reported occasional pain in his left shoulder and rare episode of shortness of breath.

The urgent thoracoabdominal CT with ivcontrast evidenced a large breach of the diaphragm with intrathoracic organ displacement. Herniation involved the stomach, spleen, bowel and pancreas (Figure 3).

The next day the patient underwent a surgical reduction of the herniated organs, there was no ischemia and the diaphragmatic disruption was repaired. Five days later the patient was discharged home.

\section{Discussion}

It is very difficult, if not impossible, to ascertain the true incidence of delayed diaphrag-
Correspondence: Ciro Paolillo, Emergency Department, Udine University Hospital, piazzale Santa Maria della Misericordia 15, 33100 Udine, Italy.

Tel: +39.0432 .552360 .

E-mail: ciropaolillo@gmail.com

Key words: Abdominal pain; Thoracic trauma; Diaphragmatic injury.

Received for publication: 20 March 2016.

Revision received: 1 July 2016.

Accepted for publication: 4 July 2016.

This work is licensed under a Creative Commons Attribution 4.0 License (by-nc 4.0).

(C) Copyright C. Paolillo and V. Siragusa, 2016

Licensee PAGEPress, Italy

Emergency Care Journal 2016; 12:5892

doi:10.4081/ecj.2016.5892

matic injury. Road traffic collisions are the most frequent causes of acquired diaphragmatic hernia, usually direct impact depresses the side of the rib cage, and can cause a tear in the diaphragm rib attachments, and even the transverse rupture of the diaphragm. In some cases acute diaphragmatic lacerations are repaired immediately, in other they are not recognised only to become apparent in the future, and some will never be detected. A delay in presentation could be explained by various different hypotheses: specific signs and symptoms may not be present during the acute phase, traumatic diaphragmatic tears are difficult to detect until there is herniation of intra-abdominal structure into the thorax. The development of symptomatic delayed diaphragmatic hernia can be a physiological consequence of the negative pressure in the chest that pulls intraabdominal contents through the diaphragmatic defect, while positive intraabdominal pressure presses these contents in the same direction. Almost $88 \%$ of the patients with delayed diaphragmatic rupture presented with complications between 9 and 12 months after trauma. Singh ${ }^{4}$ reported a diaphragmatic rupture presenting 50 years after the traumatic event. Unfortunately the physical examination is often not helpful. In the latent phase clinical exam will either be entirely normal, or there will be subtle signs. A delayed rupture may be asymptomatic or produce only mild, nonspecific symptoms, such as vague abdominal pain, chest or shoulder pain or recurrent dyspnoea for months or years. Pain may worsen with meals, it can be mistaken for peptic ulcer disease, or pain may radiate to the left shoulder, a symptom that can be mistaken for myocardial ischemia.

Physical signs are of little aid unless the diagnosis is suspected, and usually only in retrospect the signs and the physical findings can 
be related. Traumatic diaphragmatic hernias represent a diagnostic challenge not only clinically but also radiographically. Despite technical limitations, chest radiography is still used as the first step for the detection of diaphragmatic injury. Adeguate visualisation of the diaphragm can be difficult because it is thin, has a domed contour, and is contagious with soft tissue of the abdomen. Intrathoracic air in a silhouette consistent with supra-diaphragmatic viscera may occur in up to $50 \%$ of

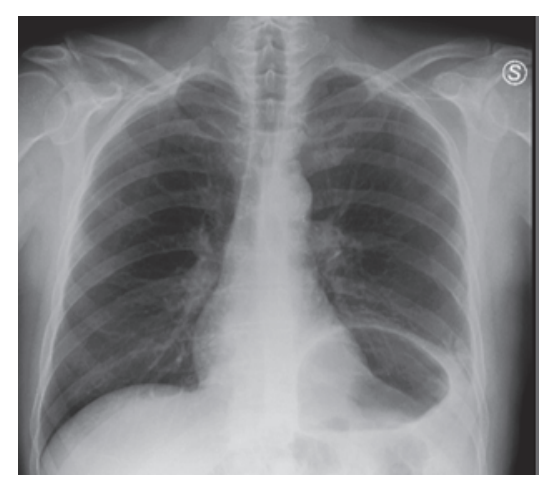

Figure 1. Anteroposterior chest X-ray on presentation (note the presence of air fluid level at the left lung base).

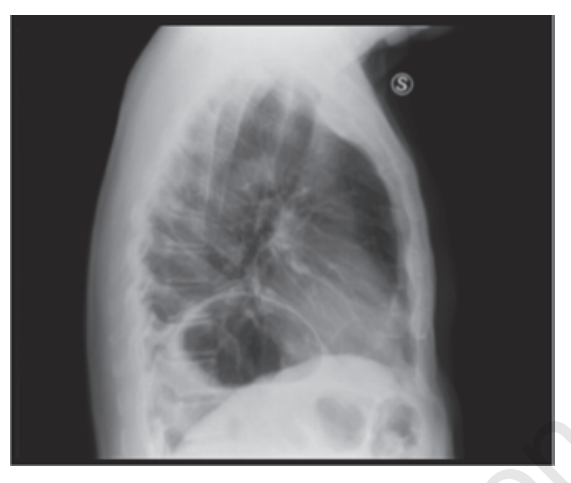

Figure 2. Latero-lateral chest X-ray. patients with left-sided diaphragmatic herniation. Murray in $1998^{5}$ reported that $62 \%$ of the patients with acute diaphragmatic injuries had a normal chest radiograph at the time of injury. $\mathrm{Nau}^{6}$ showed that only 25 to $49 \%$ of initial chest $\mathrm{X}$ rays were of diagnostic value. Frequently, a herniation at the costophrenic angle may be misdiagnosed as a pleural effusion or haemothorax on the chest radiography. As these findings are nonspecific, the trauma history in combination with unexplained radiographic findings should lead to a prompt CT. The most common findings on CT include a localised defect of the diaphragm, the absent diaphragm sign, and herniation of hollow organs into the hemithorax. Grillo in $2000,{ }^{7}$ reported that the sensitivity of a conventional CT scan detecting traumatic diaphragmatic injury ranges between 14 and $82 \%$ while specificity ranges between 76 and 100\%. Accuracy is augmented if there is a herniation of the intraabdominal contents.

\section{Conclusions}

Delayed diaphragmatic injury is a diagnos-

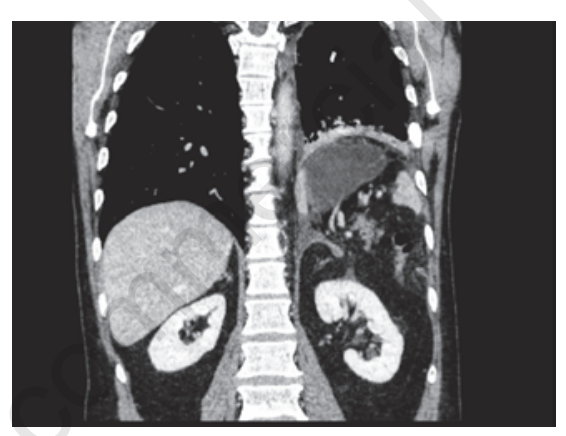

Figure 3. Coronal computed tomography scan showing disrupted left hemidiaphragm. tic challenge. The ability to make the diagnosis has somewhat improved because of the technologic advances in CT, however, it remains true that the best tool to guide the clinician toward the appropriate diagnosis is a high index of suspicion whenever there is a history of high velocity trauma, regardless of how remote, and particularly when other signs of severe trauma were present at time of injury. The morbidity and mortality of surgical intervention increases dramatically in patients presenting with acute obstructive symptoms from delayed diaphragmatic injury, whereby the need to pursue the diagnosis if there is the suspect.

\section{References}

1. Kelly J, Condon ET, Kirwan WO, Redmond HP. Post-traumatic tension faecopneumothorax in a young male: case report. World J Emerg Surg 2008;3:20.

2. Grimes OF. Traumatic injuries of the diaphragm. Diaphragmatic hernia. Am J Surg 1974;128:175-81.

3. Walchalk LR, Stanfield SC. Delayed presentation of traumatic diaphragmatic rupture. J Emerg Med 2010;39:21-4.

4. Singh S, Kalan MM, MOreyra CE, Buckman RF. Diaphragmatic rupture presenting 50 years after the traumatic event. J Trauma 2000:49:156-9.

5. Murray JA, Berne J, Asensio JA. Penetrating thoracoabdominal trauma. Emerg Med Clin North Am 1998;16:107-28.

6. Nau T, Seltz H, Mousavi M, Vecsei V. The diagnostic dilemma of traumatic rupture of the diaphragm. Surg Endosc 2001;15:9 92-6.

7. Grillo IA, Jataniah SA, Bayoumi AH, et al. Traumatic diaphragmatic hernia: an Asir region (Saudi Arabia) experience. Indian J Chest Dis Allied Sci 2000;42:9-14. 